\title{
ON THE STRUCTURE OF IDEALS OF THE DUAL ALGEBRA OF A COALGEBRA
}

BY

\author{
DAVID E. RADFORD
}

\begin{abstract}
The weak-* topology is seen to play an important role in the study of various finiteness conditions one may place on a coalgebra $C$ and its dual algebra $C *$. Here we examine the interplay between the topology and the structure of ideals of $C^{*}$. The basic theory has been worked out for the commutative and almost connected cases (see [2]). Our basic tool for reducing to the almost connected case is the classical technique of lifting idempotents. Any orthogonal set of idempotents modulo a closed ideal of $\operatorname{Rad} C *$ can be lifted. This technique is particularly effective when $C=C_{1}$. The strongest results we obtain concern ideals of $C_{1}^{*}$. Using the properties of idempotents we show that $C_{1}=$ $\Sigma_{x, y} C_{x} \wedge C_{y}$ where $C_{x}$ and $C_{y}$ run over the simple subcoalgebras of $C$. Our first theorem states that a coalgebra $C$ is locally finite and $C_{0}$ is reflexive if and only if every cofinite ideal of $C^{*}$ contains a finitely generated dense ideal. We show in general that a cofinite ideal $I$ which contains a finitely generated dense ideal is not closed. (In fact either equivalent condition of the theorem does not imply $C$ reflexive.) The preceding statement is true if $C=C_{1}$, or more importantly if $I \supset \operatorname{Rad} C^{*}$ and $C^{*} / I$ is algebraic. The second theorem characterizes the closure of an ideal with cofinite radical which also contains a finitely generated dense ideal.
\end{abstract}

In this paper we examine some of the basic aspects of the relationship between the weak-* topology and the structure of ideals of the dual algebra of a coalgebra over a field. In [2] and [4] the weak-* topology is seen to play an important role in the study of various finiteness conditions that one may place on a coalgebra. Here we try to put the topological ideas developed in these two references into a more general context.

Regarding a coalgebra $C$ as a $C$-bicomodule we turn it into a left $C$ $=C \otimes C^{\text {op }}$-comodule. This makes $C^{*}$ a left $\Rightarrow C^{*}$-module.The cyclic submodules are the closures of the principal (two-sided) ideals of $C^{*}$. Thus $C^{*}$-submodules are ideals, and the finitely generated submodules are the closed ideals of $C^{*}$ which contain a finitely generated dense ideal (see [2]). If $O M$ is a cofinite maximal ideal of $C^{*}$ or more generally an algebraic ideal which contains the Jacobson radical $\operatorname{Rad} C^{*}$, then $O M$ is a $C^{*}$-submodule (see [4]). A minor theme of this paper is the connection between the ideals and $C^{*}$-submodules.

If all ideals of the $C^{*}$ are closed then $C$ must be of finite type. For any coalgebra $C$ the finite-dimensional rational $C^{*}$-modules are those which have closed cofinite annihilator. If $C$ is reflexive (all cofinite ideals closed) then $C$ is locally finite and $C_{0}$ is reflexive (see [2]). We show that the latter condition is

Presented to the Society, April 21, 1973 under the title Ideals and the weak-* topology of the dual algebra of a coalgebra over a field $k$; received by the editors February 5, 1973.

AMS (MOS) subject classifications (1970). Primary 16A66; Secondary 16A32, 16A24, 16A21.

Key words and phrases. Coalgebra, structure of ideals, idempotents, weak-* topology. 
equivalent to saying that all cofinite $C^{*}$-submodules of $C^{*}$ are closed, or that each cofinite ideal contains a finitely generated dense ideal.

To require $C$ to be locally finite and $C_{0}$ reflexive is not enough to insure that $C$ is reflexive unless $C=C_{1}$. If $C=C_{1}$ then a cofinite ideal $I$ of $C^{*}$ which contains a finitely generated dense ideal must be closed. This is not the case in general, even if $C=C_{2}$.

The classical technique of lifting idempotents seems to be a very fruitful way of studying $C_{1}$. We show that for a coalgebra $C$ any orthogonal set of idempotents modulo a closed ideal of $\operatorname{Rad} C^{*}$ can be lifted. Cofinite ideals under fairly general circumstances contain nonzero idempotents. Our most useful result is when $C=C_{1}$ and $e^{2}=e \in C^{*}$, the $(e)$ is a closed ideal provided $e$ (restricted to $C_{0}$ ) is central.

For a coalgebra $C$ write $C_{0}=\amalg C_{x}$ as the direct sum of simple coalgebras. By the methods cited above we show $C_{1}=\Sigma_{x, y} C_{x} \wedge C_{y}$ (see also [7]).

In $\$ 4$ we characterize the closure of certain ideals in terms of $C P=\operatorname{Rad} C^{*}$. We first show that an ideal $I$ which contains a finitely generated dense ideal and such that $\operatorname{Rad}\left(C^{*} / I\right)$ is cofinite is closed if $C / I^{\perp}$ is a semisimple $C$-comodule. From this we show that the closure of an ideal $I$ which contains a finitely generated dense ideal such that $\operatorname{Rad}\left(C^{*} / I\right)$ is cofinite is $\mathcal{L} \cdot I$.

Throughout this paper we shall freely use the notation and results of [2] and [4].

\section{Preliminaries.}

1.1. C* as a topological space. For convenience we isolate those topological results found in [2] which will be needed here.

Let $V$ be a vector space. Throughout this paper we shall regard $V^{*}$ as a topological space with the weak-* topology. The closed subspaces are the annihilators $W^{\perp}$ of subspaces $W$ of $V$. For a subspace $I$ of $V^{*}$ let $I^{\perp}$ denote the annihilator of $I$ in $V$; an important observation is that $\bar{I}=I^{\perp \perp}$. We say that a subspace $J$ contained in $I$ is dense (in $I$ ) if $\bar{J}=\bar{I}$.

Let $f: V \rightarrow W$ be linear. Then $f^{*}: W^{*} \rightarrow V^{*}$ is continuous, and more importantly,

1.1.1. $f^{*}: W^{*} \rightarrow V^{*}$ is linearly closed, meaning $f^{*}(I)$ is closed whenever $I$ is a closed subspace of $W^{*}$.

Suppose $I, J \subset W^{*}$ are subspaces such that $\bar{I}=\bar{J}$. Since $f^{*}$ is continuous $\overline{f^{*}(I)}=\overline{f^{*}(J)}$. Combining this observation with 1.1.1 we have

\subsection{2. $f^{*}(\bar{I})=\overline{f^{*}(I)}$ for any subspace $I$ of $W^{*}$.}

Let $N$ be a left $C$-comodule. Then $N^{*}$ is a left $C^{*}$-module. For a fixed $n^{*} \in N^{*}$ the map $L_{n^{*}}: C^{*} \rightarrow N^{*}$ defined by $L_{n^{*}}\left(c^{*}\right)=c^{*} n^{*}$ is continuous and linearly closed $\left(L_{n^{*}}=f^{*}\right.$ where $f: N \rightarrow C$ is defined by $\left.f(n)=\sum n_{(1)}\left\langle n^{*}, \underline{n}_{(2)}\right\rangle\right)$. Since the sum of closed subspaces is closed, finitely generated submodules of $N^{*}$ are closed.

The focus of this whole discussion is the following basic example.

1.1.3. Let $C$ be a coalgebra and $C^{\text {op }}$ denote the coalgebra $C$ with "twisted" 
structure, set $C=C \otimes C^{\text {op }}$. Then $(C, \omega)$ is a left $C$-module $\left(\omega(c)=\sum c_{(1)} \otimes c_{(3)}\right.$ $\left.\otimes c_{(2)} \in C \otimes C\right)$. Now $A=C^{*} \otimes C^{* o p}$ is a dense subalgebra of $C^{*}$ and the module action on $C^{*}$ restricted to $A$ is determined by $a \otimes b \cdot c^{*}=a c^{*} b$ for $a \otimes b \in \mathcal{A}$ and $c^{*} \in C^{*}$. Thus, by 1.1.2,

1.1.4. $C^{*} \cdot a=\overline{A \cdot a}=\overline{(a)}$, where $(a)$ is the principal (two-sided) ideal generated by $a \in C^{*}$.

This means that $C^{*}$-submodules of $C^{*}$ are ideals, and that closed ideals of $C^{*}$ are $C^{*}$-submodules.

Furthermore (1.3.5 of [2]),

1.1.5. the finitely generated $C^{*}$-modules of $C^{*}$ are the closed ideals which contain a finitely generated dense ideal.

1.2. Semisimple $C^{*}$-modules. The ideas in this section are of relevance primarily to $\S 4$.

For a coalgebra $C$ let $R(C)=C / C_{0}^{+}$where $C_{0}^{+}=C_{0} \cap$ ker $\varepsilon$. Then $R(C)$ is connected, and moreover the coradical filtration of each is related in a natural way. For if $\pi: C \rightarrow R(C)$ is the natural projection then

1.2.1. $\pi\left(C_{n}\right)=R(C)_{n}$ for all $n \geq 0$.

It is shown in [2] that $C_{0}^{\perp}=\operatorname{Rad} C^{*}$, the Jacobson radical of $C^{*}$. Thus,

1.2.2. $R(C)^{*}=\operatorname{Rad} C^{*} \oplus k \cdot \varepsilon$.

Notation. If $C$ is a coalgebra and $C=C \otimes C^{\text {op }}$, set $\mathcal{J}=\operatorname{Rad} C^{*}$ and $C R$ $=R(C)^{*}$.

1.2.3. Lemma. Let $N$ be a simple left $C$-comodule. Then $N$ is a D-comodule for some simple subcoalgebra $D$ of $C$.

Proof. Since $N$ is simple, $N=n<C^{*}$ for any fixed $0 \neq n \in N$. But $I_{n}$ (the annihilator of $n$ ) is a cofinite maximal right ideal of $C^{*}$. Thus the annihilator $O M$ of $N$ is a cofinite primitive (hence maximal) ideal of $C^{*}$. Let $D=\propto M^{\perp}$. Q.E.D.

For a left $C$-comodule $N$ let $N_{0}$ denote the sum of the simple subcomodules of $N$.

1.2.4. Lemma. Let $N$ be a left $C$-comodule and $J=\operatorname{Rad} C^{*}$. Then $N_{0}=\{n \in N: n$ $<J=(0)\}$, so $N_{0}=\left(J \cdot N^{*}\right)^{\perp}$.

Proof. $M=\{n \in N: n<J=(0)\}$ is a submodule of $N$, in fact a $C_{0^{-}}$ comodule since $J^{\perp}=C_{0}$. By 14.0.1, p. 288 of [6], $M \subset N_{0}$. By 1.2.3, $N_{0} \subset M$. The rest is clear. Q.E.D.

For a left $C^{*}$-module $M$ let $\operatorname{Rad} M$ denote the intersection of all maximal submodules of $M$.

1.2.5. Proposition. Suppose $N$ is a left $C$-comodule and $N^{*}$ is a finitely generated $C^{*}$-module. If $J=\operatorname{Rad} C^{*}$, then

(1) $\operatorname{Rad} N^{*}=J \cdot N^{*}$ and

(2) $0 \rightarrow J \cdot N^{*} \rightarrow N^{*} \rightarrow N_{0}^{*} \rightarrow 0$ is exact.

Proof. $J \cdot N^{*}$ is closed since $J$ is a closed ideal and $N^{*}$ is finitely generated. Thus $J \cdot N^{*}=\left(J \cdot N^{*}\right)^{\perp \perp}=N_{0}^{\perp}$. The result is now immediate. Q.E.D. 
1.2.6. Corollary. Suppose $N$ is a left $C$-comodule and $M$ is a finitely generated submodule of $N^{*}$. Then $\operatorname{Rad} M=J \cdot M$.

Proof. $M$ is closed, so the projection $N \rightarrow N / M^{\perp}=Q$ induces an inclusion of $C^{*}$-modules $Q^{*} \rightarrow{ }^{u} N^{*}$ with $u\left(Q^{*}\right)=M$. By the previous proposition $\operatorname{Rad} M$ $=u\left(\operatorname{Rad} Q^{*}\right)=u\left(J \cdot Q^{*}\right)=J \cdot M$. Q.E.D.

2. Ideals and $C^{*}$-submodules. In this section we examine the relationship between ideals and the $C^{*}$-submodules of $C^{*}$. It turns out that the classical technique of lifting idempotents is particularly useful in this regard when $C=C_{1}$.

2.1. $C$ as a $C$-comodule. In Example 1.1.3, we introduced the left $C=C \otimes C^{\text {op }}$ comodule action on a coalgebra $C$. If $N$ is a $C$-bicomodule (a left and right $C$ comodule with commuting actions) then the map $\omega: N \rightarrow C \otimes N\left(\omega(n)=\sum n_{(1)}\right.$ $\left.\otimes n_{(3)} \otimes n_{(2)}\right)$ turns $N$ into a left $C$-comodule; and the subbicomodules ( $C^{*}$ subbimodules) are the left $C$-subcomodules. The following statements are immediate.

2.1.1. The category of left $C$-comodules and the category of $C$-bicomodules are isomorphic.

2.1.2. The subcoalgebras of $C$ ( $C^{*}$-subbimodules) are the $C$-subcomodules.

2.1.3. $\Delta: C \rightarrow C\left(\Delta c=\sum c_{(1)} \otimes c_{(2)}\right)$ is a map of $C$-comodules.

2.1.4. Suppose $\pi: C \rightarrow D$ is a map of coalgebras. Then $\Pi=\pi \otimes \pi: C$ $\rightarrow D\left(D=D \otimes D^{\text {op }}\right)$ is a map of coalgebras. Thus (by pushout) $C$ is a left $D$ comodule. The algebra map $\Pi^{*}: D^{*} \rightarrow C^{*}$ induces (by pull-back) a left $D^{*}$-module structure on $C^{*}$. This is the transpose of the $D$-comodule structure on $C$.

3.17 of [4] gives the first important connection between ideals and $C^{*}$ modules.

For an ideal $I$ of $C^{*}$ let $\operatorname{Rad}(I)$ denote the intersection of all maximal ideals lying above $I$. Recall that a $k$-algebra $A$ is called algebraic if $k[a]$ is finite dimensional for all $a \in A$, and we call an ideal $I$ of $A$ algebraic if $A / I$ is an algebraic algebra.

2.1.5. Proposition. (3.17 of [4]). Suppose $C$ is any coalgebra and I an algebraic ideal of $C^{*}$ containing $\operatorname{Rad} C^{*}$. Then $I$ is a $C^{*}$-module.

The following elementary observation is useful.

2.1.6. Suppose $C$ is a coalgebra and $I$ is a closed ideal of $C^{*}$. Then $\operatorname{Rad}(I)$ is closed.

Proof. If $D=I^{\perp}$ then $0 \rightarrow I \rightarrow C^{*} \rightarrow^{u} D^{*} \rightarrow 0$ is exact, where $u$ is the restriction map. It is clear that $\operatorname{Rad}(I)=u^{-1}\left(\operatorname{Rad} D^{*}\right)$. But $\operatorname{Rad} D^{*}$ is closed, thus $\operatorname{Rad}(I)$ is also since $u$ is continuous. Q.E.D.

2.1.7. Proposition. Suppose $I$ is an ideal of $C^{*}$ which contains a finitely generated dense ideal. If all maximal ideals which contain $I$ are algebraic, then $\operatorname{Rad}(I)$ is closed.

Proof. Suppose $\left(a_{1}, \ldots, a_{n}\right)$ is dense in $I$. Then $\bar{I}=c^{*} \cdot a_{1}+\cdots+c^{*} \cdot a_{n}$ 
$\subset C^{*} \cdot I \subset \bar{I}$ implies $\bar{I}=C^{*} \cdot I$. If $\circlearrowleft M$ is a maximal ideal which contains $I$, then $\bar{I}=C^{*} \cdot I \subset$ o $M$ by 2.1.5. Thus $\operatorname{Rad}(I)=\operatorname{Rad}(\bar{I})$ and the conclusion follows by 2.1.6. Q.E.D.

2.1.8. Proposition. Suppose I contains a finitely generated dense ideal. Then I is dense (in $C^{*}$ ) if and only if no proper algebraic ideal contains $I$.

Proof. $(\Rightarrow)$ If $I \subset J$ where $J$ is a proper algebraic ideal, then there is a maximal ideal $O M$ lying over $J$, and $O M$ is necessarily algebraic. Thus $\bar{I}=C^{*} \cdot I \subset \mathcal{} M$ by 2.1.5, so $\bar{I} \neq C^{*}$.

$(\Leftrightarrow)$ Let $D=I^{\perp}$. Then we have the exact sequence $0 \rightarrow \bar{I} \rightarrow C^{*} \rightarrow D^{*} \rightarrow 0$. Thus $D=(0)$; otherwise there is a proper cofinite maximal ideal lying over $I$. Q.E.D.

In $\$ 2.2$, we will show that when $C=C_{1}$ a cofinite ideal of $C^{*}$ contains a cofinite $C^{*}$-submodule under fairly general circumstances. An essential step of the argument depends on Corollary 2.1.10 of the following statement:

2.1.9. Proposition. Suppose $\pi: C \rightarrow D$ is a surjective map of coalgebras with ker $\pi$ finite dimensional. Then every cofinite ideal of $C^{*}$ contains a cofinite $C^{*}$-submodule if and only if every cofinite ideal of $D^{*}$ contains a cofinite $D^{*}$-submodule.

Proof. The induced algebra map $u=\pi^{*}: D^{*} \rightarrow C^{*}$ is injective and $A$ $=\pi^{*}\left(D^{*}\right)$ is a cofinite subalgebra of $C^{*}$.

$\Leftrightarrow$ Suppose $I$ is a cofinite ideal of $D^{*}$. Then $u(I)$ is a cofinite ideal of $A$. Thus $M=C^{*} / u(I)$ is a finite-dimensional left $A$-module, so $J C^{*} \subset u(I)$ for some cofinite ideal $J$ of $A$. But $J C^{*}$ is a cofinite right ideal of $C^{*}$; thus the annihilator $L$ of the right $C^{*}$-module $C^{*} / J C^{*}$ is a cofinite ideal of $C^{*}$ and $L \subset J C^{*} \subset u(I)$. By assumption there is a cofinite $C^{*}$-module $K \subset L$. Therefore $u^{-1}(K)$ is a $D^{*}$ submodule of $D^{*}$ and $u^{-1}(K) \subset u^{-1}(u(I))=I$.

$(\Leftrightarrow)$ Let $I$ be a cofinite ideal of $C^{*}$. Then $J=u^{-1}(I)$ is a cofinite ideal of $D^{*}$. By assumption there is a cofinite $D^{*}$-submodule $J_{1} \subset J$. Since $u$ is also a map of $D^{*}$-modules by $2.1 .4, I_{1}=u\left(J_{1}\right)$ is a cofinite $D^{*}$-submodule of $C^{*}$. Now $\Delta^{*}: C^{*} \rightarrow C^{*}$ is a map of $C^{*}$-modules by 2.1 .3 , hence a map of $D^{*}$-modules. Therefore $\gamma_{1}=\Delta^{*-1}\left(I_{1}\right)$ is a cofinite $D^{*}$-submodule of $C^{*}$. Since $A=u\left(D^{*}\right)$ has finite codimension, the dense subalgebra $C^{*} \otimes C^{* \text { op }}$ of $C^{*}$ is a finitely generated left $D^{*} \otimes D^{* 0 p}$. module. Therefore, by 2.1.4 again, $C^{*}$ is a finitely generated left $D^{*}$-module. Now $M=C^{*} / \gamma_{1}$ is a finite-dimensional left $C^{*}$-module, so $\mathcal{L} C^{*} \subset \gamma_{1}$ for some cofinite ideal $\mathcal{L}$ of $D^{*}$. By 1.3.8 of [3], the right ideal $\mathcal{L} C^{*}$ of $C^{*}$ is cofinite. Hence there is a cofinite ideal $\gamma$ of $C^{*}$ (see the preceding paragraph) with $\gamma \subset \mathcal{L} C^{*} . \Delta^{*}(\gamma)$ is a cofinite $C^{*}$-submodule of $C^{*}$, and $\Delta^{*}(\gamma) \subset \Delta^{*}\left(\gamma_{1}\right) \subset I_{1}=u\left(J_{1}\right) \subset I$. Q.E.D.

2.1.10. Corollary. Suppose $C=C_{1}$ and is almost connected. Then every cofinite ideal of C* contains a cofinite C*-submodule.

Proof. The projection $C \rightarrow^{\pi} R(C)$ is surjection and ker $\pi=C_{0}^{+}$is finite dimensional. If $J=\operatorname{Rad} C^{*}$ then $J^{2}=(0)$. By $1.2 .2, R(C)^{*}=J \oplus k \cdot 1$ so $R(C)^{*}$ is commutative. 
The result follows easily from 1.1.4 and the preceding proposition, since principal left ideals are closed. Q.E.D.

2.2. On lifting idempotents. Let $C$ be any coalgebra. We first show that any orthogonal set of idempotents modulo a closed ideal $I \subset \operatorname{Rad} C^{*}$ can be lifted to an orthogonal set of idempotents.

2.2.1. Lemma. Suppose $C$ is a finite-dimensional coalgebra, $D$ a subcoalgebra with $C_{0} \subset D$. If $\left\{e_{1}, \ldots, e_{n}\right\} \subset D^{*}$ is an orthogonal set of nonzero idempotents, there exists an orthogonal set of idempotents $\left\{u_{1}, \ldots, u_{n}\right\} \subset C^{*}$ with $u_{i} \equiv e_{i}$ on $D$ for all $1 \leq i \leq n$.

Proof. Let $I=D^{\perp}$. Then $I \subset C_{0}^{\perp}=\operatorname{Rad} C^{*}$. We have the exact sequence $0 \rightarrow I \rightarrow C^{*} \rightarrow^{u} D^{*} \rightarrow 0$ where $u$ is the restriction map. Choose $a_{1}, \ldots, a_{n}$ $\in C^{*}$ with $u\left(a_{i}\right)=e_{i}$ for all $1 \leq i \leq n$. Then $\left\{a_{1}, \ldots, a_{n}\right\}$ is an orthogonal set of nonzero idempotents modulo $I$, so, by Proposition 2 on p. 73 of [1], there exists an orthogonal set of idempotents $u_{1}, \ldots, u_{n} \in C^{*}$ with $u_{i}-a_{i} \in I$, i.e., $u_{i} \equiv e_{i}$ on $D$ for all $1 \leq i \leq n$. Q.E.D.

2.2.2. Proposition. Let $C$ be any coalgebra and $D$ a subcoalgebra with $D \supset C_{0}$. If $\left\{e_{\alpha}\right\} \subset D^{*}$ is an orthogonal set of idempotents then there exists an orthogonal set of idempotents $\left\{u_{\alpha}\right\} \subset C^{*}$ with $u_{\alpha} \equiv e_{\alpha}$ on $D$ for all $\alpha$.

Proof. Let $\Im$ be the family of all pairs $\left(E,\left\{v_{\alpha}\right\}\right)$ where $E$ is a subcoalgebra such that $D \subset E$, and $\left\{v_{\alpha}\right\} \subset E^{*}$ is an orthogonal set of idempotents satisfying $v_{\alpha} \equiv e_{\alpha}$

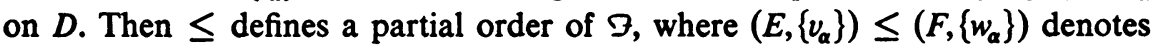
$E \subset F$ and $w_{\alpha} \equiv v_{\alpha}$ on $E$ all $\alpha$. Zorn's lemma applies; thus there is a maximal element $\left(E,\left\{w_{\alpha}\right\}\right) \in \mathcal{Y}$.

Suppose $F$ is a finite-dimensional subcoalgebra of $C$. Then $F_{0}=C_{0} \cap F$ $\subset E \cap F$. Since nonzero orthogonal idempotents are independent, the set $\left\{v_{\alpha}^{\prime}\right\}$ has only finitely many nonzero elements $\left(v_{\alpha}^{\prime}\right.$ is $v_{\alpha}$ restricted to $\left.E \cap F\right)$. By 2.2.1, there exists an orthogonal set of idempotents $\left\{w_{\alpha}\right\} \subset F^{*}$ with $w_{\alpha} \equiv v_{\alpha}$ on $E \cap F$. It is easy to see that the rule $u_{\alpha}(e+f)=v_{\alpha}(e)+w_{\alpha}(f)$ defines a functional $u_{\alpha} \in(E+F)^{*}$ for all $\alpha$ and that $\left(E+F,\left\{u_{\alpha}\right\}\right) \in \Im$. This implies $E=C$. Q.E.D.

An immediate consequence of 2.2 .2 is the following:

2.2.3. Let $C$ be a coalgebra, $D$ a subcoalgebra such that $C_{0} \subset D$, and $e^{2}=e \in D^{*}$. There exists an idempotent $u \in C^{*}$ with $u \equiv e$ on $D$.

2.2.4. Corollary. Suppose $C$ is a coalgebra and $I$ is a cofinite ideal of $C^{*}$ with $\operatorname{Rad}(I)$ closed. There is an idempotent $e \in I$ which is central (when restricted to $C_{0}$ ) and such that $\operatorname{Rad}(I)=\operatorname{Rad}((e))$.

Proof. We have the exact sequence $0 \rightarrow \operatorname{Rad} C^{*} \rightarrow C^{*} \rightarrow^{4} C_{0}^{*} \rightarrow 0$ where $u$ is the restriction map. Since $J=\operatorname{Rad}(I)$ is closed, $u(J)$ is closed. By 3.5.1 of [2], $u(\operatorname{Rad}(I))=(f)$ for some central idempotent $f \in C_{0}^{*}$. By 2.2.3, there is an idempotent $e \in C^{*}$ with $u(e)=f$. Thus $e \in J=u^{-1}(u(J))$, and since $J^{n} \subset I$ 
some $n$, we see that $e=e^{n} \in I$. It is clear that $\operatorname{Rad}((e))=J$. Q.E.D.

2.2.5. Remark. Suppose $\left\{e_{a}\right\}$ is any orthogonal set of idempotents of $C^{*}$. Then $e=\Sigma e_{\alpha}$ is defined since all but finitely many of the $e_{\alpha}$ 's vanish on a given finitedimensional subcoalgebra (nonzero orthogonal idempotents are independent). Clearly $e^{2}=e$.

2.2.6. Corollary. Let $C$ be any coalgebra, $D$ a subcoalgebra with $C_{0} \subset D$. Suppose $\left\{f_{\alpha}\right\} \subset D^{*}$ is an orthogonal set of idempotents with $\varepsilon=\sum f_{\alpha}$. There exists an orthogonal set of idempotents $\left\{e_{\alpha}\right\} \subset C^{*}$ such that $\varepsilon=\sum e_{\alpha}$ and $e_{\alpha} \equiv f_{\alpha}$ on $D$ for all $\alpha$.

Proof. By 2.2.2, there exists an orthogonal set of idempotents $\left\{e_{\alpha}^{\prime}\right\}$ with $e_{\alpha}^{\prime} \equiv f_{\alpha}$ on $D$. Fix a particular $\alpha$, say $\alpha_{0}$, and set

$$
\begin{aligned}
e_{\alpha} & =e_{\alpha}^{\prime}, & & \alpha \neq \alpha_{0}, \\
& =\varepsilon-\sum_{\alpha \neq \alpha_{0}} e_{\alpha}^{\prime}, & & \alpha=\alpha_{0} .
\end{aligned}
$$

Then $\left\{e_{\alpha}\right\}$ is an orthogonal set of idempotents which meets our criteria. Q.E.D.

Now suppose $e, f \in C^{*}$ are any idempotents and write $e+e^{\prime}=\varepsilon=f+f^{\prime}$.

2.2.7. $(e \rightarrow C<f)^{\perp}=C^{*} \cdot e^{\prime}+f^{\prime} \cdot C^{*}$, so for subspaces $V, W \subset e \rightarrow C$ $-f$ it follows that $V \wedge W \subset e \rightarrow C<f$.

Proof. That $(e \rightarrow C)^{\perp}=C^{*} \cdot e^{\prime}$ and $(C<f)^{\perp}=f^{\prime} \cdot C^{*}$ is easy to verify. So

$$
\begin{aligned}
(e \rightarrow C<f)^{\perp} & =(e \rightarrow C \cap C<f)^{\perp} \\
& =(e-C)^{\perp}+(C<f)^{\perp} \\
& =C^{*} \cdot e^{\prime}+f^{\prime} \cdot C^{*} .
\end{aligned}
$$

If $I=(e \rightarrow C<f)^{\perp}$ then $I \subset I^{2}$ since $e^{\prime}$ and $f^{\prime}$ are idempotents. Therefore,

$$
(e \rightarrow C<f) \wedge(e \rightarrow C<f)=\left(I^{2}\right)^{\perp} \subset I^{\perp}=e \rightarrow C<f \text {. Q.E.D. }
$$

2.2.8. If $D, E$ are subcoalgebras of $C$ and $c \in D \wedge E$ then

$$
\Delta e \rightarrow c<f \in C<f \otimes e \rightarrow E+D<f \otimes e \rightarrow C .
$$

Proof. For $a, b \in C^{*}$ we have $\Delta a \rightarrow c<b=\Sigma c_{(1)}<b \otimes a \rightarrow c_{(2)}$, so

$$
\begin{aligned}
\Delta e \rightarrow c<f & \in(C \otimes E+D \otimes C) \cap C<f \otimes e \rightarrow C \\
= & C-f \otimes e \rightarrow E+D<f \otimes e \rightarrow C . \text { Q.E.D. }
\end{aligned}
$$

2.2.9. Lemma. If $e \rightarrow C_{0}<e$ is a subcoalgebra then so is $e \rightarrow C_{1}<e$; in fact, $e \rightarrow C_{1}<e=\left(e \rightarrow C_{0}<e\right) \wedge\left(e \rightarrow C_{0}<e\right)$.

Proof. If $e \rightarrow C_{0}<e$ is a subcoalgebra,then $e \rightarrow C_{0}=e \rightarrow C_{0}<e=C_{0}$ $-e$ since $C_{0}$ is the direct sum of simple coalgebras. By 2.2.7, $\left(e \rightarrow C_{0}\right)$ 
$\wedge\left(C_{0}<e\right) \subset(e \rightarrow C<e) \cap C_{1}=e \rightarrow C_{1}<e$. By 2.2.8, e $\rightarrow C_{1}<e \subset(e$ $\left.\rightarrow C_{0}\right) \wedge\left(C_{0}-e\right)$. Q.E.D.

2.2.10. Corollary. If $e \rightarrow C_{0}<e=f \rightarrow C_{0}<f$ and is a coalgebra, then $e \rightarrow C_{1}$ $<e=f \rightarrow C_{1}<f$.

Remark. If $e \rightarrow C_{1}<e$ is a coalgebra we cannot conclude that $e \rightarrow C_{2}<e$ is also in general. The smallest possible example demonstrating this must be at least five dimensional. One such is the following:

Let $C$ have basis $1, z, v, w$, and $x$ and define

$$
\begin{array}{rlrl}
\Delta 1 & =1 \otimes 1, & \\
\Delta z & =z \otimes z, & & \varepsilon(1)=1=\varepsilon(z), \\
\Delta v & =v \otimes z+1 \otimes v, & \varepsilon(v)=\varepsilon(w)=\varepsilon(x)=0 . \\
\Delta w & =w \otimes 1+z \otimes w, & & \\
\Delta x & =x \otimes 1+v \otimes w+1 \otimes x, &
\end{array}
$$

If $e(1)=1, e(z)=e(v)=e(w)=e(x)=0$ then $e^{2}=e$ and $e \rightarrow C<e$ is spanned by 1 and $x$. See also Example 3.4.

2.2.11. Let $C$ be a coalgebra and write $C_{0}=\amalg C_{x}$, where $C_{x}$ is simple for all $x$. Then $C_{1}=\Sigma_{x, y} C_{x} \wedge C_{y}$.

Proof. For each $x$ we can find in $C_{0}^{*}$ idempotents $\varepsilon_{x}$ such that $\varepsilon_{x} \rightarrow C_{0}=C_{x}$ $=C_{0}<\varepsilon_{x} .\left\{\varepsilon_{x}\right\}$ is orthogonal and $\sum \varepsilon_{x}=\varepsilon$. By 2.2.6, we can find an orthogonal set of idempotents $\left\{e_{x}\right\} \subset C^{*}$ such that $\sum e_{x}=\epsilon$ and $e_{x} \equiv \epsilon_{x}$ on $C_{0}$. Notice

$$
C=\bigoplus_{x, y} e_{x} \rightarrow C<e_{y} .
$$

If $c \in e_{x} \rightarrow C<e_{y}$ then $c=e_{x} \rightarrow c<e_{y}$, so if $c \in C_{1}$ in addition, by 2.2.8, $\Delta c \in C \otimes e_{x} \rightarrow C_{0}+C_{0}<e_{y} \otimes C$. Therefore $c \in C_{y} \wedge C_{x}$. Q.E.D.

Remarks. Suppose in 2.2.11, $C=C_{1}$ and is pointed. Write $C_{0}=k(X)$ and set $C_{x}=k \cdot x$ for $x \in X$. If $x \neq y$ and $c \in e_{x} \rightarrow C<e_{y}$ then one sees that $\Delta c=c \otimes x+y \otimes c$, and $e_{x} \rightarrow C<e_{y}=\{c \in C: \Delta c=c \otimes x+y \otimes c\}$. This gives the characterization of pointed coalgebras $C=C_{1}$ discovered by Taft and Wilson (see [7]).

Combining 2.2.9 with 2.2.7 we have

2.2.12. Let $C=C_{1}$ and $e^{2}=e \in C^{*}$ such that $e \rightarrow C_{0}<e$ is a coalgebra. Then $(e)$ is closed; in fact $(e)=C^{*} \cdot e+e \cdot C^{*}$.

Now suppose $C$ is a coalgebra and $\operatorname{Rad} C^{*}$ is nil. If $I$ is an ideal such that $\operatorname{Rad}(I)$ is closed, by Proposition 1 on p. 72 of [1] and 3.5.1 of [2], there is an idempotent $e \in I$ which is central (when restricted to $C_{0}$ ) and such that $\operatorname{Rad}(I)=\operatorname{Rad}((e))$. Let $D=(e)^{\perp}$. If $C=C_{1}$, we have the exact sequence ( $u$ the restriction map) 


$$
0 \rightarrow(e)=C^{*} \cdot e+e \cdot C^{*} \rightarrow C^{*} \stackrel{\mu}{\rightarrow} D^{*} \rightarrow 0
$$

and $u(I) \subset \operatorname{Rad} D^{*}$.

2.2.13. Proposition. Suppose $C=C_{1}$ is a coalgebra, and I a cofinite ideal with $\operatorname{Rad}(I)$ closed. Then I contains a cofinite C*-submodule.

Proof. $D=D_{1}$ since $C=C_{1}$ for any subcoalgebra $D$ of $C$. The result follows immediately from (*) and 2.1.10 since $u$ is a map of $C^{*}$-modules. Q.E.D.

2.2.14. Lemma. Let $C$ be any coalgebra, $I \subset \operatorname{Rad} C^{*}$ an ideal. If $I^{2}+V$ is a dense subspace of $I$, then $C^{*} \cdot V$ is a left ideal dense in $I$.

Proof. Let $D$ be a finite-dimensional subcoalgebra and $u$ : $C^{*} \rightarrow D^{*}$ the restriction map. It suffices to show $u\left(C^{*} \cdot V\right)=u(I)$. But $u(I)=u\left(I^{2}+V\right)$ $=u(I)^{2}+u\left(C^{*} \cdot V\right)$. Thus we may assume $C=D$ and $I^{2}+C^{*} \cdot V=I$. By induction, $I^{n}+C^{*} \cdot V=I$ for all $n$. Since $I$ is nilpotent, $C^{*} \cdot V=I$. Q.E.D.

2.2.15. Corollary. Suppose $I \subset \operatorname{Rad} C^{*}$ is an ideal and $I^{2}+V$ is dense in $I$ for some finite-dimensional $V$. Then $I^{n}$ is finitely generated as a left ideal (hence closed) and a cofinite subspace of $I$ for all $n$.

Proof. By 2.2.14, the closed left ideal $C^{*} \cdot V$ is dense in $I$; thus $C^{*} \cdot V=I$. Suppose $a_{1}, \ldots, a_{r}$ span $V$. Then $I^{2}=I a_{1}+\cdots+I a_{r}$ implies that $I$ is a finitely generated left ideal of $A=I \oplus k \cdot \varepsilon$. The rest follows by 1.1.1 of [2]. Q.E.D.

It should be noted that if $C=C_{n}$ for some $n$ then the ideal in 2.2 .15 is in fact finite dimensional.

2.2.16. Proposition. Suppose $C=C_{1}$ and $I$ is an ideal of $C^{*}$ such that $\operatorname{Rad}(I)$ is closed. If $I^{2}+V$ is dense in I for some finite-dimensional $V$, then $I=C^{*} \cdot a_{1}+\cdots$ $+C^{*} \cdot a_{n}+b \cdot C^{*}$ for some $a_{1}, \ldots, a_{n}, b \in I$.

Proof. Consider (*). $u(I)$ satisfies the hypothesis of the proposition. By 2.2.15, $u(I)$ is finite dimensional. The conclusion now quickly follows. Q.E.D.

2.2.17. Proposition. Suppose $C=C_{1}$ and $I$ is an ideal of $C^{*}$ with $\operatorname{Rad}(I)$ cofinite. If $I$ contains a finitely generated dense ideal then $I=C^{*} \cdot a_{1}+\cdots+C^{*} \cdot a_{n}$ $+b \cdot C^{*}$ for some $a_{1}, \ldots, a_{n}, b \in C^{*}$.

Proof. By 2.1.7, $\operatorname{Rad}(I)$ is closed. First assume $C$ is almost connected and $I \subset \operatorname{Rad} C^{*}$. Let $A=\operatorname{Rad} C^{*} \oplus k \cdot \varepsilon$. Since $\operatorname{dim}\left(C^{*} / A\right)<\infty$ the dense ideal $L$ of $I$ is generated as an ideal of $A$ by $a_{1}, \ldots, a_{n}$ say. Since $A$ is commutative $L=A \cdot a_{1}+\cdots+A \cdot a_{r}=\bar{L}$ which implies $L=I$. Now we use (*) to complete the proof. Q.E.D.

We can generalize 2.2.16 to any coalgebra. The conclusion, however, will not be quite as sharp.

2.2.18. Proposition. Let $C$ be any coalgebra and $I$ an ideal of $C^{*}$ with $\operatorname{Rad}(I)$ closed. If $I^{2}+V$ is dense in $I$ for some finite-dimensional $V$, then $I^{n}$ contains a finitely generated dense ideal and $\overline{I^{n}}$ is a cofinite subspace of $\bar{I}$ for all $n$. 
Proof. By 3.5.1. of [2] and 2.2.3, $J=\operatorname{Rad}(I)=\operatorname{Rad}(I)=\operatorname{Rad}((e))$ for some idempotent $e$. If $D=(e)^{\perp}$ we have the exact sequence $0 \rightarrow \overline{(e)} \rightarrow C^{*} \rightarrow^{u} D^{*}$ $\rightarrow 0$, where $u$ is the restriction map, and $u(J)=\operatorname{Rad} D^{*}$. But $e \in J$ implies $e \in \cap J^{n} \subset \bar{I}$, so $\overline{(e)} \subset \overline{I^{n}}=\overline{I^{n}}$ for all $n$. By $2.2 .14, \overline{I^{n}}=u^{-1}\left(u\left(I^{n}\right)\right)$ is a cofinite subspace of $I=u^{-1}(u(I))$. The remaining part will follow once we observe that if $a \in I$ and $u(a)=u(e)$ then $\overline{(e)} \subset \overline{\left(a^{n}\right)}$ for all $n$. Q.E.D.

If $I \subset C^{*}$ is an ideal then $\operatorname{Rad}(\bar{I})$ is closed by 2.1.6. Thus

2.2.19. Corollary. Suppose $C$ is any coalgebra, $I$ an ideal of $C^{*}$ such that $I^{2}+V$ is dense in I for some finite-dimensional $V$. Then $\overline{I^{n}}$ is an ideal cofinite in $\bar{I}$ for all $n$.

3. Locally finite coalgebras. It has been shown in [2] that $C$ reflexive implies $C$ is locally finite and the coradical $C_{0}$ is reflexive. The converse is true in the commutative case, but even the hypothesis that the second term of the coradical filtration $C_{1}$ is reflexive does not as much imply that $C_{2}$ is reflexive in general. We find in this section a topological description of locally finite coalgebras with $C_{0}$ reflexive which gives the commutative theorem as a special case. First we need partial generalization of 3.2.1 of [1].

3.1. Lemma. Suppose $C=C_{1}$ and is almost connected. If all cofinite C*-modules of $C^{*}$ are closed, then $C$ is finite dimensional.

Proof. All maximal ideals of $C^{*}$ are closed since $\operatorname{Rad} C^{*}=C_{0}^{\perp}$ is cofinite. Since cofinite $C^{*}$-modules are closed by assumption, by 2.1 .12 every cofinite ideal of $C^{*}$ is closed. Thus $C$ is reflexive. By 3.2.1 of [2], $C$ is finite dimensional. Q.E.D.

3.2. Theorem. Let $C$ be any coalgebra. Then the following are equivalent:

(1) $C_{0}$ is reflexive and $C$ is locally finite.

(2) All cofinite $C^{*}$-modules of $C^{*}$ are closed.

(3) Every cofinite ideal of $C^{*}$ contains a finitely generated dense ideal.

Proof. (1) $\Rightarrow(2)$. Let $I$ be a cofinite $C^{*}$-module of $C^{*}$. Then $\operatorname{Rad}(I)=J$ is closed since $C_{0}$ is reflexive; so if $C^{*} \rightarrow^{\mu} C_{0}^{*}$ is the projection we conclude $u(J)=(u(a))$ for some $a \in I$. Let $D=(a)^{\perp}$. Then $D_{0}=J^{\perp}$ and is therefore finite dimensional. Since $C$ is locally finite $D_{1}$ is finite dimensional, and this implies $D$ is strongly reflexive by 4.1 .1 of [2]. Since $C^{*} \cdot a=\overline{(a)}$ we have the exact sequence $0 \rightarrow C^{*} \cdot a \rightarrow C^{*} \rightarrow D^{*} \rightarrow 0$. But $\pi(I)$ is finitely generated as a left ideal of $D^{*}$ so, by $2.1 .4, I=\pi^{-1}(\pi(I))$ is a finitely generated $C^{*}$-module. This means that $I$ is closed.

(2) $\Rightarrow(3)$. Let $I$ be a cofinite ideal of $C^{*}$. Then $C^{*} \cdot I=E^{\perp}$ where $E$ is a finitedimensional subcoalgebra of $C$. If $D=E_{0} \wedge E_{0}$, then $D_{0}=E_{0}$ and, using 2.1.4, we see the cofinite $D^{*}$-modules of $D^{*}$ are closed. By $3.1, D=D_{1}$ is finite dimensional. Since $D \wedge D \subset \wedge^{n} D_{0}$ some $n$ we have that $D \wedge D$ is finite dimensional by 4.2 .2 of [2]. By the same theorem $C^{*} \cdot I$ is a finitely generated submodule. It is easy to find generators in $I$. Since $C^{*} \cdot I=\bar{I}$, we are done.

(3) $\Rightarrow(1)$. By 2.1 .5 all cofinite maximal ideals of $C^{*}$ are closed, so $C_{0}$ is reflexive 
by 3.5.3 of [2]. By 4.2.2 of [2], $C$ is locally finite. Q.E.D.

If $C=C_{1}$ we can make a much stronger statement.

3.3. Corollary. Suppose $C=C_{1}$. Then the following are equivalent:

(1) $C_{0}$ is reflexive and $C$ is locally finite.

(2) $C$ is reflexive.

(3) If I is a cofinite ideal of $C^{*}$, then $I=C^{*} \cdot a_{1}+\cdots+C^{*} \cdot a_{n}+b \cdot C^{*}$ for some $a_{1}, \ldots, a_{n}, b \in I$.

Proof. (1) $\Leftrightarrow$ (3) follows by 3.2 and 2.2.17.

(3) $\Rightarrow(2)$ is clear since principal left (or right) ideals of $C^{*}$ are closed.

$(2) \Rightarrow(1)$ is clear. Q.E.D.

Remarks. (1) If $C$ is commutative, then $C^{*} \cdot a=(a)$ so Theorem 3.2 gives the essence of 5.1.1 of [2].

(2) If $C_{0}$ is reflexive (which one may reasonably expect for infinite fields $k$; see $\$ 3.7$ of [2]) then 3.2 gives a topological formulation of local finiteness. $C_{0}$ reflexive and $C$ locally finite does not necessarily imply $C_{2}$ reflexive. This means 3.3 cannot be improved in general.

3.4. Example. Let $k$ be an infinite field, and let $N$ denote the set of positive integers. For $1<n \in N$ choose vector spaces $V(n) \simeq k^{n} \simeq W(n)$, set $V$ $=\bigoplus V(n), W=\bigoplus_{n} W(n)$ and $V \cdot W=\bigoplus_{n} V(n) \otimes W(n)$ (let $v \cdot w=v \otimes w$ for $v \in V(n)$ and $w \in W(n)$ ). Endow $C=N \oplus V \oplus W \oplus V \cdot W$ with the coalgebra structure determined by

$$
\begin{aligned}
& \Delta n=n \otimes n, \\
& \varepsilon(n)=1 \text { for all } m \in N \text {, } \\
& \Delta v=v \otimes n+1 \otimes v, \\
& \varepsilon(v)=0, \\
& \Delta w=w \otimes 1+n \otimes w, \\
& \varepsilon(w)=0, \\
& \Delta v \cdot w=v \cdot w \otimes 1+v \otimes w+1 \otimes v \cdot w, \quad \varepsilon(v \cdot w)=0 \text { for all } v \in V(n) \\
& \text { and } w \in W(n) \text {. }
\end{aligned}
$$

One can check easily that $C_{0}=k^{(N)}$, so is reflexive by 3.6 of [4]; and that $C_{1}=N \oplus V \oplus W$ and is locally finite, so $C$ is also.

Let $M=1^{\perp}$. Then $M$ is a maximal ideal of $C^{*}$. The functionals $M^{2}$ restricted to $V \cdot W$ is a proper dense subspace. Thus $M^{2} \subsetneq \mathcal{}, M$ and $\triangle M^{2}$ is not closed. Since $C^{*}=k \cdot \varepsilon \oplus \mathcal{}$, any subspace $I$ satisfying $\triangle M^{2} \subset I \subset \subset$ is an ideal. Therefore there are cofinite ideals $I$ which are not closed satisfying $\mathcal{M}^{2} \subset I \subset \mathcal{}$. This means $C$ is not reflexive.

Remarks. In the preceding example $k \cdot 1 \wedge k \cdot 1=k \cdot 1$. Suppose $e \in C^{*}$ is any idempotent satisfying $e(n)=1-\delta_{1, n}$. Then

(1) $\overline{(e)}=\alpha$, so $(e)$ is not closed since $(e) \subset M^{2}$;

(2) if $\delta M^{2} \subset I \subset \mathcal{}$, then $e \in I$; therefore $\overline{(e)}=\bar{I}$. Thus a cofinite ideal which contains a finitely generated dense ideal is not necessarily closed.

4. The closure of an ideal. Example 3.4 shows that in general a cofinite ideal of $C^{*}$ which contains a finitely generated dense ideal is not closed. Proposition 4.3 
gives a condition under which such an ideal is closed. From this result we derive the main theorem of this section, which concerns the role Rad C* plays in the description of the closure of a ideal.

The first lemma is a special case of 4.3.

4.1. Lemma. Suppose $C$ is connected and $I$ is an ideal of $C^{*}$ which contains a finitely generated dense ideal. If $C / I^{\perp}$ is a semisimple C-comodule, then $I$ is finite dimensional.

Proof. We have the commutative diagram

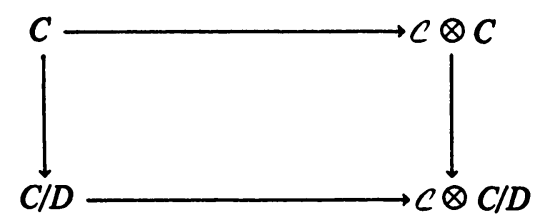

where $D=I^{\perp}$. If $G(C)=\{1\}$ then $C_{0}=k \cdot 1 \otimes 1$. By $1.2 .3, C / D$ is in fact a $C_{0^{-}}$ comodule. Thus if $(C / D, \omega)$ is the underlying $C$-comodule structure on $C / D$, then $\omega \overline{(c)}=1 \otimes 1 \otimes \bar{c}$ for all $c \in C$. This means $\Sigma c_{(1)} \otimes c_{(3)} \otimes c_{(2)}-1 \otimes 1 \otimes c$ $\in \operatorname{ker} \pi=C \otimes C \otimes D$ or

$$
\sum c_{(1)} \otimes c_{(2)} \otimes c_{(3)}-1 \otimes c \otimes 1 \in C \otimes D \otimes C \text { for } c \in C .
$$

If $f \in I$ then $f(D)=(0)$; so, given $a, b \in C^{*}$,

$$
\langle a * f * b, c\rangle=\langle a * b, 1\rangle\langle f, c\rangle \text { for each } c \in C
$$

which implies $a * f * b=\langle a * b, 1\rangle f$. Therefore any subspace of $I$ is an ideal, so $I$ is finite dimensional by assumption. Q.E.D.

To reduce 4.3 to the connected case we will need the following technical lemma.

4.2. Lemma. Let $D$ be connected and $\pi: C \rightarrow D$ a surjective coalgebra map with $\pi\left(C_{0}\right)=D_{0}$. If $E$ is a subcoalgebra of $C$ and $C / E$ is a semisimple C-comodule then $D / \pi(E)$ is a semisimple D-comodule.

Proof. We have the array

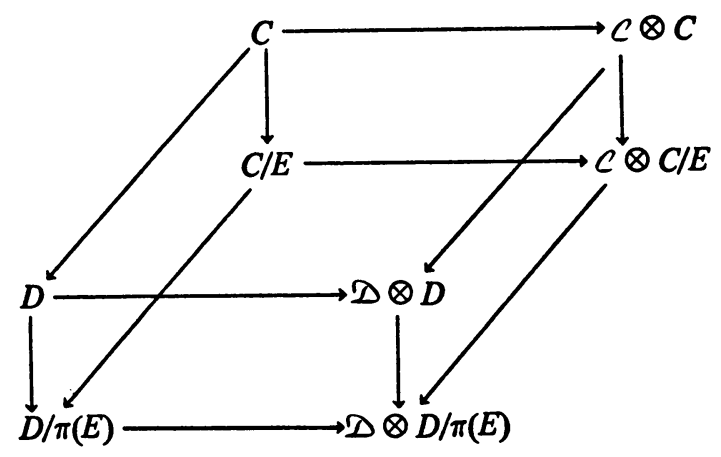


with commuting top and sides, hence the bottom diagram commutes. Now $C_{0} \subset C_{0} \otimes C_{0}$ so if $\Pi: C \rightarrow D$ is the induced coalgebra map, $\Pi\left(C_{0}\right) \subset \pi\left(C_{0}\right)$ $\otimes \pi\left(C_{0}\right)=D_{0}$ since $D$ is connected. Therefore $\Pi^{*}\left(\operatorname{Rad} D^{*}\right) \subset \operatorname{Rad} C^{*}$. Using the bottom of the above array we have the commutative diagram

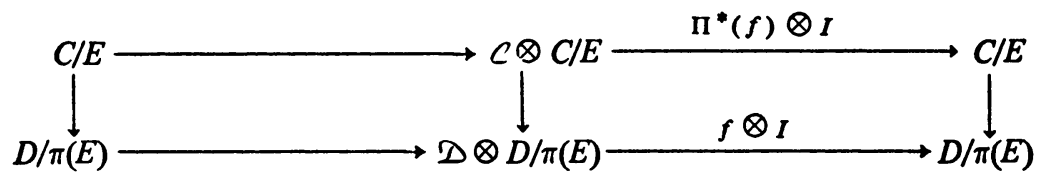

for any $f \in D^{*}$. Therefore, if $f \in \operatorname{Rad} D^{*}$, by 1.2.4, $\bar{d}<f=0$ for all $d \in D$. This means again by 1.2 .4 that $D / \pi(E)$ is semisimple. Q.E.D.

4.3. Proposition. Let $C$ be a coalgebra, $I$ an ideal of $C^{*}$ with $\operatorname{Rad}(I)$ cofinite and which contains a finitely generated dense ideal. If $C / I^{\perp}$ is a semisimple C-comodule, then $I$ is closed.

Proof. Let $C \rightarrow C / D=N$ be the projection where $D=I^{\perp}$. Write $C_{0}=E_{1}$ $\oplus D_{0}$. Since $N$ is semisimple, $\pi\left(E_{1}\right) \oplus M=N$ for some subcomodule $M$ of $N$. By 2.1.2, $E_{2}=\pi^{-1}(M)$ is a subcoalgebra of $C$, and

$$
E_{1} \cap E_{2}=E_{1} \cap D=E_{1} \cap D_{0}=(0) \text {. }
$$

Therefore $C^{*}=E_{1}^{*} \times E_{2}^{*}$. Let $u: C^{*} \rightarrow E_{2}^{*}$ be the restriction map. Now $J$ $=\operatorname{Rad}(I)$ is closed by 2.1 .7 so ker $u \subset J$ since $J^{\perp}=D_{0} \subset E_{2}$. From this it is easy to see that ker $u \subset I$. Therefore $I=u^{-1}(u(I))$. Clearly $u(I)$ satisfies the hypothesis of the proposition. The map $E_{2} \rightarrow^{\pi} C / D$ is a map of comodules; therefore $M=\pi\left(E_{2}\right)$ is a semisimple $\varepsilon_{2}$-comodule. Hence to prove the proposition we may assume $C=E_{2}$. But also notice $u(I)^{\perp}=I^{\perp}=D$ and

$$
\left(E_{2}\right)_{0}=C_{0} \cap E_{2}=\left(E_{1} \oplus D_{0}\right) \cap E_{2}=D_{0} \cap E_{2}=D_{0} .
$$

Thus

4.4. To prove 4.3 we may assume in addition that $C$ is almost connected and $I \subset \operatorname{Rad} C^{*}$.

Now assume the additional hypothesis. If we can reduce to the connected case we will be done by 4.1 .

Let $\bar{C}=R(C)=C / C_{0}^{+}$and $C \rightarrow^{\circ} \bar{C}$ be the projection. Suppose $\rho^{*}=f: \bar{C}^{*}$ $\rightarrow C^{*}$ is the induced algebra map; set $\gamma=f^{-1}(I)$ and $\mathcal{L}=f^{-1}(L)$ where $L \subset I$ is a finitely generated ideal dense in $I$. Then $I=f(\gamma)$ and $L=f(\mathcal{L})$ since $f\left(\bar{C}^{*}\right)=\operatorname{Rad} C^{*} \oplus k \cdot 1$. Now $f$ is continuous and linearly closed so

$$
f(\overline{\mathcal{L}})=\overline{f(\mathcal{L})}=\bar{L}=\bar{I}=f(\bar{\gamma})
$$

which implies that $\mathcal{L}$ is dense in $\gamma$. Since $\operatorname{dim}\left(C^{*} / \bar{C}^{*}\right)<\infty$ we have $C^{*} \otimes C^{* \text { op }}$ is a finitely generated left $\bar{C}^{*} \otimes \bar{C}^{* o p}$-module. This means $\gamma$ is a finitely generated 
ideal of $\bar{C}^{*}$. To show $I=f(\gamma)$ is closed we need only show that $\gamma$ is closed. Since $\rho\left(I^{\perp}\right)=\gamma^{\perp}$ by $4.2, \bar{C} / \gamma^{\perp}$ is a semisimple $\bar{C}$-comodule. Thus we may assume $C=\bar{C}=R(C)$ and the reduction to the connected case is complete. Q.E.D.

Now we come to the main result of this section. The proof is a reduction to the previous proposition.

4.5. Theorem. Suppose $C$ is a coalgebra, I an ideal of $C^{*}$ with $\operatorname{Rad}(I)$ cofinite and which contains a finitely generated dense ideal. Then $\mathcal{O} \cdot I=I$.

Proof. The projection $\pi: C \rightarrow C / I^{\perp}=N$ is a surjection of $C$-comodules since $I^{\perp}$ is a subcoalgebra. Since $\pi^{*}\left(N^{*}\right)=I$ we see that $N^{*}$ is a finitely generated $C^{*}$. module since $I$ contains a finitely generated dense ideal.

Set $D=\pi^{-1}\left(N_{0}\right)$. Then $D$ is a subcoalgebra of $C$.

We derive from the commutative diagram

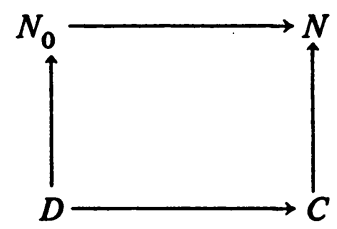

the commutative diagram

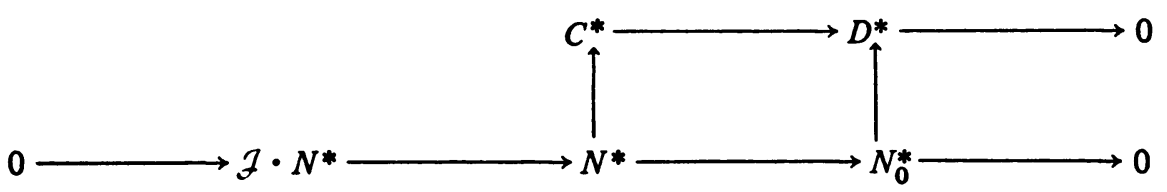

with bottom row exact by 1.2 .5 . Since $\mathcal{J} \cdot I=\mathcal{J} \cdot\left(C^{*} \cdot I\right)=\mathcal{J} \cdot \bar{I}$ we have the commutative diagram

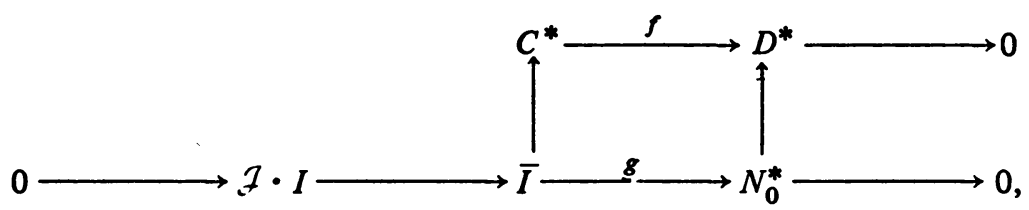

where $f$ is the restriction map.

Now $\mathcal{R} \cdot I=g^{-1}(g(\bar{I}))$. Therefore to show $\mathcal{R} \cdot I=\bar{I}$ we need only show $g(I)=g(I)$, or equivalently $f(I)=f(I)$. Since $f$ is continuous and linearly closed $f(\bar{I})=\overline{f(I)}$. Thus it suffices to show that $f(I)$ is closed. It is clear $f(I)$ is an ideal of $D^{*}$ which satisfies the hypotheses. of the theorem. Thus we may assume $C=D$. Observe $N_{0}=\pi(D)=D / I^{\perp}$ is a semisimple $D$-comodule, since $\pi: D \rightarrow N_{0}$ is a surjective $C$-comodule map. Since $f(I)^{\perp}=I^{\perp}$ we are reduced to Proposition 4.3. Q.E.D.

4.6. Corollary. Let I be a cofinite ideal of $C^{*}$ which contains a finitely generated dense ideal. Then $\bar{I}=u_{1} \cdot I+\cdots+u_{\gamma} \cdot I$ for some units $u_{1}, \ldots, u_{\gamma}$ of $\propto \mathcal{R}$. 
For any ideal $I$ of $C^{*}$ let $I$ denote the sum of the $\subset$-submodules of $I$. Then for $a \in I$ the computation $\mathcal{R} \cdot(a)=(a)+\mathcal{J} \cdot a$ shows that $I$ is also an ideal.

4.7. Corollary. Let I be a cofinite ideal of $C^{*}$ which contains a finitely generated dense ideal. Then $I$ is closed if and only if $\operatorname{Rad}(\underline{I})$ is cofinite and closed.

Proof. $(\Rightarrow)$ This is clear since $\operatorname{Rad}(I)=\operatorname{Rad}(I)$ is closed in this case by 2.1.7.

$\Leftrightarrow$ Suppose $\operatorname{Rad}(I)$ is cofinite and closed. Let $u: C^{*} \rightarrow C_{0}^{*}$ be the projection. Then $u(I)=u(\operatorname{Rad}(I))$, so $u(I)=u((a))$ for some $a \in I$. But $\operatorname{Rad}((a))=\operatorname{Rad}(I)$ which implies $\overline{(a)}=\mathcal{L} \cdot(a) \subset I$ by 4.5 . Let $D=(a)^{\perp}$. Then $D_{0}=\operatorname{Rad}(I)^{\perp}$ is finite dimensional and $0 \rightarrow \subset \mathcal{R} \cdot(a) \rightarrow C^{*} \rightarrow^{*} D^{*} \rightarrow 0$ is exact. $D$ is reflexive (see 4.2.6 of [2]) so $I=\pi^{-1}(\pi(I))$ is closed. Q.E.D.

\section{REFERENCES}

1. J. Lambek, Lectures on rings and modules, Blaisdell, Waltham, Mass., 1966. MR 34 \#5857.

2 . R. G. Heyneman and D. E. Radford, Reflexivity and coalgebras of finite type, J. Algebra 28 (1974), 215-246.

3. R. G. Heyneman and M. E. Sweedler, Affine Hopf algebras. I, J. Algebra 13 (1969), 192-241. MR 39 \#6876.

4. D. E. Radford, Coreflexive coalgebras, J. Algebra 26 (1973), 512-535.

5. J. R. Sullivan, The uniqueness of integrals for Hopf algebras and some existence theorems of integrals for commutative Hopf algebras, J. Algebra 19 (1971), 426-440.

6. M. E. Sweedler, Hopf algebras, Math. Lecture Note Series, Benjamin, New York, 1969. MR 40 \#5705.

7. E. J. Taft and R. L. Wilson, On antipodes in pointed Hopf algebras (to appear).

DePARTMENT OF MATHEMATICS, LAWRENCE UNIVERSTTY, APPLETON, WisConsin 54911 